\title{
Charge Ordering and Polaron Formation in the Magnetoresistive Oxide $\mathrm{La}_{0.7} \mathrm{Ca}_{0.3} \mathrm{MnO}_{3}$
}

\author{
C. P. Adams,,$^{1,2}$ J. W. Lynn, ${ }^{1,2}$ Y. M. Mukovskii, ${ }^{3}$ A. A. Arsenov, ${ }^{3}$ and D. A. Shulyatev ${ }^{3}$ \\ ${ }^{1}$ NIST Center for Neutron Research, National Institute \\ of Standards and Technology, Gaithersburg, MD 20899-8562 \\ ${ }^{2}$ Department of Physics, University of Maryland, \\ College Park, MD 20742 \\ ${ }^{3}$ Moscow Steel and Alloys Institute, Moscow 117936, Russia
}

(November 11, 2018)

\begin{abstract}
Neutron scattering has been used to study the nature of the ferromagnetic transition in a single crystal of the perovskite $\mathrm{La}_{0.7} \mathrm{Ca}_{0.3} \mathrm{MnO}_{3}$. Diffuse scattering from lattice polarons develops as the Curie temperature is approached from below, along with short range polaron correlations that are consistent with stripe formation. Both the scattering due to the polaron correlations and the anomalous quasielastic component in the magnetic fluctuation spectrum maximize very close to $T_{C}$, in a manner remarkably similar to the resistivity, indicating that they have a common origin.
\end{abstract}

75.30.Vn, 71.38.+i, 75.30.Kz, 75.30.Ds

One of the simplifying features of conventional isotropic ferromagnets such as $\mathrm{Fe}, \mathrm{Co}, \mathrm{Ni}$, and $\mathrm{EuO}$ is the isolation of the spin system from the underlying lattice. This made these materials ideal candidates to investigate the low temperature magnetic excitations, critical dynamics, scaling behavior, and nature of secondorder phase transitions. This situation contrasts dramatically with the colossal magnetoresistive (CMR) oxides, exemplified by the ferromagnetic doping regime of $\mathrm{La}_{1-x} \mathrm{Ca}_{x} \mathrm{MnO}_{3}(0.15<x<0.5)$ [1]. The transport in these perovskites is directly connected to the magnetic system through the double exchange mechanism, while the Jahn-Teller distortion of the $\mathrm{Mn}^{3+} \mathrm{O}_{6}$ couples the magnetic and lattice systems, resulting in the electronic, lattice, and magnetic degrees of freedom being intimately intertwined [2,3]. The ground state spin dynamics has a number of unusual properties such as large linewidths and anomalous dispersion [4,5], while the combined metal-insulator and ferromagnetic transition has been found to be quite different from conventional isotropic ferromagnets 6 8]. In particular, the spin wave stiffness does not collapse as $T \rightarrow T_{C}$, but instead a quasielastic diffusive component develops in the excitation spectrum. Above $T_{C}$, the conductivity is characterized by hopping that is believed to be associated with polarons [1],6] 9-11, and recently direct evidence for the formation of lattice polarons has been observed in single crystals of the layered manganite $\mathrm{La}_{1.2} \mathrm{Sr}_{1.8} \mathrm{Mn}_{2} \mathrm{O}_{7}$ [12], and in the cubic, half-doped $\left(\mathrm{Nd}_{0.12} \mathrm{Sm}_{0.88}\right)_{0.52} \mathrm{Sr}_{0.48} \mathrm{MnO}_{3}$ material [13]. However, the relationship between the ferromagnetic transition and the polaron formation associated with the metal-insulator transition has remained a mystery. In the present work we observe the formation of lattice polarons in an optimally-doped cubic CMR material, and find clear evidence for polaron ordering in the paramagnetic phase that is consistent with some models of stripe formation. More importantly, the temperature dependence of this polaron intensity develops simultaneously with the quasielastic spin fluctuation scattering, directly connecting these two phenomena with the resistivity.

The sample is a $0.7 \mathrm{~g}$ single crystal grown by the floating zone technique [14], with a single-peaked mosaic less than $0.25^{\circ}$. At this composition the crystal structure is orthorhombic, but the distortion is small and the domains are equally populated. Therefore for simplicity we will employ cubic notation $(a=3.867 \AA$ at room temperature), where nearest-neighbor manganese atoms are along the [100]-type directions. Most measurements were taken in the $(h k 0)$ plane on the BT-2 and BT-9 triple-axis spectrometers at NIST, using a variety of incident energies $(13.7,14.7,30.5$, and $50 \mathrm{meV}$ ) and collimations. For the unpolarized neutron measurements the monochromator and analyzer crystals were pyrolytic graphite (PG), while for polarized beam measurements Heusler alloy polarizers were employed. PG filters were used when appropriate to suppress higher-order wavelength contaminations. Statistical uncertainties quoted represent one standard deviation.

A typical example of the magnetic fluctuation spectrum observed below the Curie temperature $\left(T_{C}=\right.$ $257 \mathrm{~K}$ ) is shown in Fig. 1(a). A $q$-independent nuclear incoherent scattering of $2.6 \mathrm{cts} / \mathrm{min}$ and a flat background of $1.6 \mathrm{cts} / \mathrm{min}$ have been subtracted from these data. At this reduced wave vector of $(0.09,0,0)$ well defined spin wave excitations are observed in neutron energy gain $(E<0)$ and energy loss $(E>0)$, and the solid curve is a least-squares fit of the spin wave cross section, along with the quasielastic component centered at $E=0$, convoluted with the instrumental resolution. Data taken at a series of $q$ 's reveal that the spin waves obey the usual quadratic dispersion law $E_{S W}=\Delta+D(T) q^{2}$, with a 

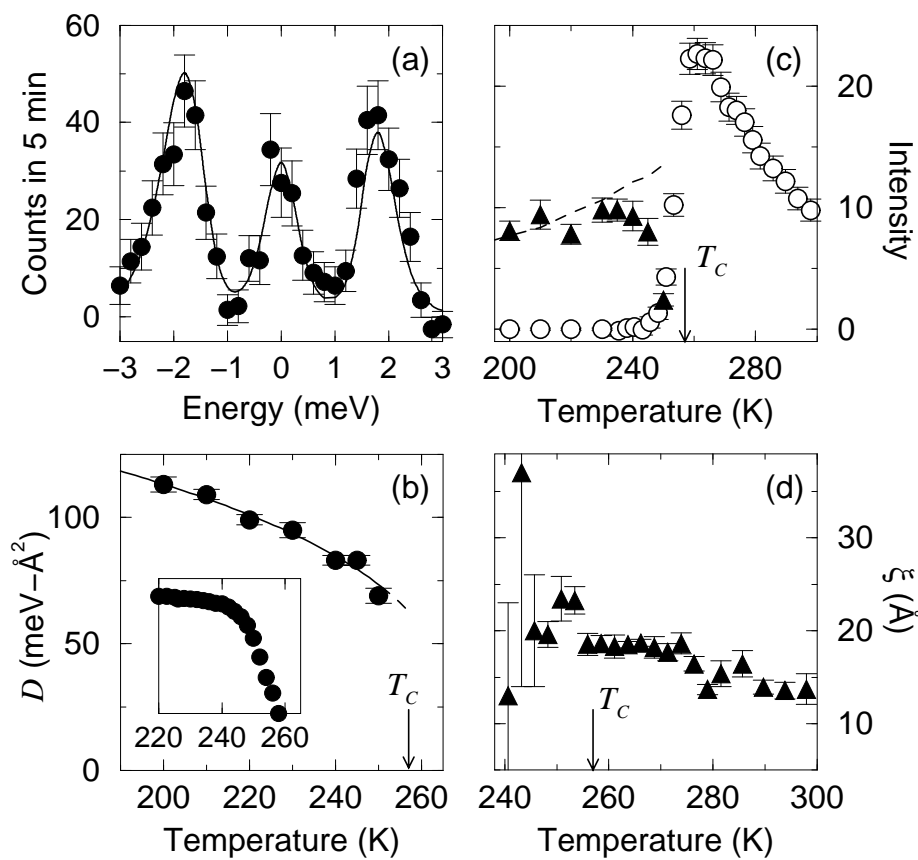

FIG. 1. (a) Constant- $Q$ scan taken at $T=240 \mathrm{~K}$ with reduced wave vector $(0.09,0,0)$, showing the spin waves and quasielastic central peak. (b) Spin wave stiffness $D(T)$, which does not appear to fall to zero at $T_{C}=257(1) \mathrm{K}$, as indicated by the relative magnetization (inset). Extinction of the (100) Bragg peak causes an unphysical flattening of the magnetization below $\sim 240 \mathrm{~K}$. (c) Intensity of spin waves (triangles) and the central peak (circles) versus temperature. The spin wave intensity follows Bose-Einstein statistics (dashed line) until the central peak develops and eventually dominates the spectrum. (d) Correlation length associated with the central peak, which is weakly temperature dependent and does not diverge at the ferromagnetic transition.

negligible spin wave gap $\Delta$, indicative of an isotropic ferromagnet, as has been found for all these CMR ferromagnets [15]. The temperature dependence of the spin stiffness coefficient $D(T)$ obtained from these fits is shown in Fig. 1(b), which can be compared with the magnetization determined from the (100) Bragg peak (inset). A well defined Curie temperature of $T_{C}=257(1) \mathrm{K}$ is obtained, and there does not appear to be any significant distribution of $T_{C}$ 's in the sample. The spin wave stiffness does not seem to collapse at $T_{C}$, but instead we see the development of the quasielastic component as shown in Fig. 1(a). The energy width of this scattering is quadratic in wave vector, with a spin diffusion constant $\Lambda=15(7) \mathrm{meV}-\AA^{2}$ (in this symmetry direction). The temperature evolution of this component is shown in Fig. 1(c), where we see that it attains its maximum intensity close to $T_{C}$. The spin wave intensities, on the other hand, show a decrease as the central component develops, rather than the usual increase of the Bose-Einstein population factor. Finally, Fig. 1(d) shows the correlation length obtained from the $q$-dependence of the quasielastic scattering. Below $T_{C}$ the quasielastic scattering is well separated from the spin wave contribution with this relatively good energy resolution $(0.15 \mathrm{meV}$ FWHM), while above $T_{C}$ all the scattering is quasielastic. We find a length scale $\sim 16 \AA$ that is only weakly temperature dependent. This central peak, with the associated short length scale, has been interpreted as the spin component of polarons [6,10]. All these detailed results obtained on this high quality single crystal are in good overall agreement with previous measurements on polycrystalline samples [6], as well as single crystal results on related $x=1 / 3$ doped compounds [7].

The scattering from a lattice polaron arises from the structural distortion that surrounds a carrier, and traps it. Individual polarons generate diffuse (Huang) scattering around the fundamental Bragg peaks, and we observe such diffuse scattering in the present $\mathrm{La}_{0.7} \mathrm{Ca}_{0.3} \mathrm{MnO}_{3}$ crystal, which is similar to the scattering from polaron distortions recently reported in the layered manganite $\mathrm{La}_{1.2} \mathrm{Sr}_{1.8} \mathrm{Mn}_{2} \mathrm{O}_{7}$ [12, and in the half-doped cubic $\left(\mathrm{Nd}_{0.12} \mathrm{Sm}_{0.88}\right)_{0.52} \mathrm{Sr}_{0.48} \mathrm{MnO}_{3}$ material [13]. The temperature dependence of this diffuse scattering is shown in Fig. 2 for a wave vector of $(1.85,2,0)$. The signal increases rapidly as the Curie temperature is approached from below as the polarons form, while above $T_{C}$ we observe only a weak temperature dependence. This suggests that the number of polarons increases rapidly as $T \rightarrow T_{C}$, while above $T_{C}$ the number is roughly constant.

In addition to the diffuse polaron scattering, we observe well-developed polaron-polaron correlations, which give rise to satellite peaks such as those shown in Fig. 3. This is a scan of the elastic scattering measured around the $(4,0,0)$ nuclear Bragg peak. In the paramagnetic state $(280 \mathrm{~K})$ we see two satellite peaks, indicating an ordering wave vector $\left(\frac{1}{4}, \frac{1}{4}, 0\right)$ and equivalent directions. The background scattering at $220 \mathrm{~K}$ is also shown, and has a gentle variation which is due to the proximity of an aluminum powder diffraction line from the sample holder; the lower part of the figure shows the subtraction of the

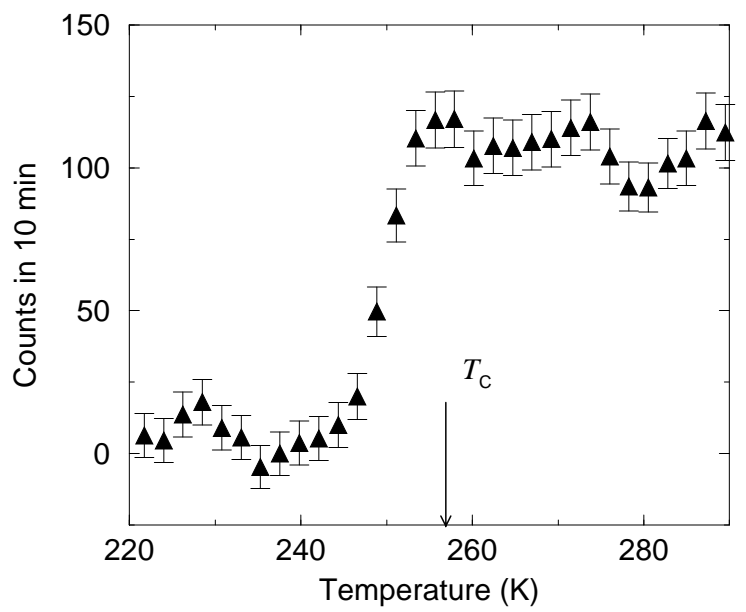

FIG. 2. The temperature dependence of the diffuse polaron scattering measured at $(1.85,2,0)$. 


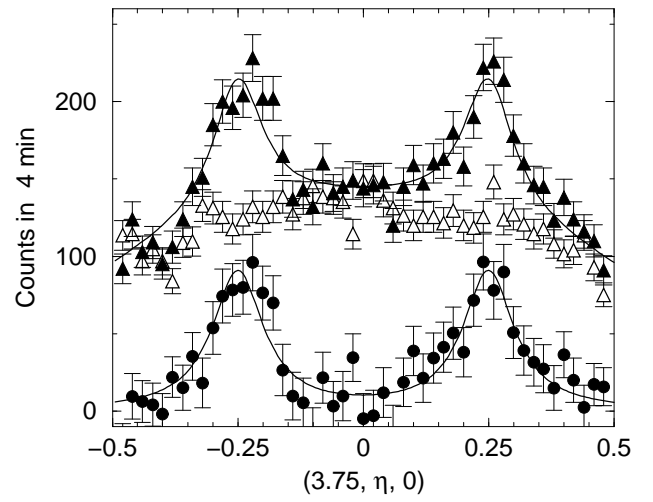

FIG. 3. Transverse elastic scans through $(3.75,0,0)$ taken at $220 \mathrm{~K}$ and $280 \mathrm{~K}$ (open and filled triangles respectively), showing the clear development of polaron peaks at $\left(\frac{1}{4}, \frac{1}{4}, 0\right)$. The subtracted data are shown as full circles. The solid curves are fits to Lorentzian peaks.

two data sets to obtain the net scattering. These polaron peaks have also been observed around other Bragg peaks such as the $(2,0,0),(3,0,0),(5,0,0),(3,1,0)$, $(4,2,0)$, and $(2,2,0)$, while the intensity around the $(0,0,0),(1,0,0),(1,1,0)$, and $(2,1,0)$ was below our detection limit. This trend for the intensity to increase with increasing $Q$ suggests that the dominant contribution to this scattering is from the lattice. This has been directly confirmed by polarized beam measurements, which show that the scattering in these peaks is dominated by the non-spin-flip (lattice) component.

A familiar model that can explain both the ordering wave vector of $\left(\frac{1}{4}, \frac{1}{4}, 0\right)$ for the lattice polaron scattering as well as the overall behavior of the observed intensities of the short-range correlation peaks is the $\mathrm{CE}$ model [1,2] developed for the half-doped case. The CE model has an orbitally ordered Jahn-Teller lattice with charge stripes in the [110] direction, and an antiferromagnetic ground state. Such ordering has been observed for $x \geq \frac{1}{2}$, with the lattice and antiferromagnetic ordering at low temperatures [17]. This contrasts with the present ferromagnetic system, where the orbital/charge correlations are short range in nature, occur only above $T_{C}$, and are not accompanied by antiferromagnetic correlations. The lattice part of the CE model would of course need to be modified to accommodate the smaller doping level, but with the same wave vector; so far there is no apparent change in the ordering wave vector with doping. In particular, in the half-doped material the diffuse signal overwhelmed any peaks in the scattering, making it difficult to identify correlations [13], but the weak structure observed suggested correlations with a wave vector of magnitude $\sim 0.3-0.35$ and in the [110] direction. In the $x=0.4$ bilayer system clear peaks were observed, but the wave vector is in the [100] direction, and of (incommensurate) magnitude 0.30 [12]. One similarity, however, is that the charge peaks for $\mathrm{La}_{0.7} \mathrm{Ca}_{0.3} \mathrm{MnO}_{3}$ and the bilayer both have a substantial intrinsic width, indicating that the polaron ordering is short range in nature. The width of the peaks is only weakly temperature dependent, and yields a correlation length of $\sim 10(2) \AA$ (Fig. 3), which is the same basic length scale observed for the quasielastic magnetic scattering [Fig. 1(d)]. The polaron peaks in both materials are also elastic, indicating that the polarons are static on a time scale of 1 ps. The polarons are surely hopping, though, and one of the interesting avenues to explore experimentally will be to investigate the nature of these peaks with much higher energy resolution, and in particular to determine if the observed $q$-widths are related to dynamics of the polarons instead of static short range order.

Stripe formation has been observed in the related cuprates and nickelates, but the intrinsic magnetism in those systems is always antiferromagnetic in nature. This gives rise to separate satellite peaks associated with the charge and spin order [18,19]. We have found no evidence for separate magnetic satellites in $\mathrm{La}_{0.7} \mathrm{Ca}_{0.3} \mathrm{MnO}_{3}$, which is not surprising in this ferromagnetic system where the spin stripes (or spin polaron correlations) would be expected to give a contribution at the same satellite positions as the charge satellites. It might then seem surprising that we observe no significant magnetic component to the satellite peaks. However, the nature of the two types of scattering is quite different. The lattice scattering originates from well-formed (static, on this time scale) Jahn-Teller distorted $\mathrm{MnO}_{6}$ octahedra, which then have a correlation length of $\sim 10 \AA$. The spin stripes would have the same short correlation length, but the magnetic scattering itself has a short correlation range of only $\sim 15 \AA$ [Fig. 1(d)]. Model calculations then show that the combination of the two short correlation ranges renders the spin stripe scattering too weak to be observed at any of the satellite positions. We conclude then that the scattering of the lattice component of the polarons occurs around the high- $Q$ fundamental Bragg peaks, along with the broad satellite peaks associated with polaron-polaron correlations, while the dominant magnetic contribution occurs around the low- $Q$ fundamental Bragg reflections, in the form of the quasielastic scattering as we now discuss.

The temperature dependence of the intensity of the satellite peak at $(3.75,0.25,0)$ is shown in Fig. 4. We see that the scattering begins to develop in this sample $\sim 30 \mathrm{~K}$ below $T_{C}$, rapidly develops as $T \rightarrow T_{C}$, and peaks just above the ordering temperature. This behavior is very similar to the temperature dependence of the resistivity, which is shown as the dashed curve in Fig. 4 (scaled to the peak in the scattering). The intensity of the quasielastic component of the spin fluctuation spectrum [measured at $(1.03,0,0)]$ has been similarly scaled and is also shown in the figure. We see that the temperature dependence of the two types of scattering through the ferromagnetic phase transition is virtually identical to the resistivity, indicating that they all have a common origin. 


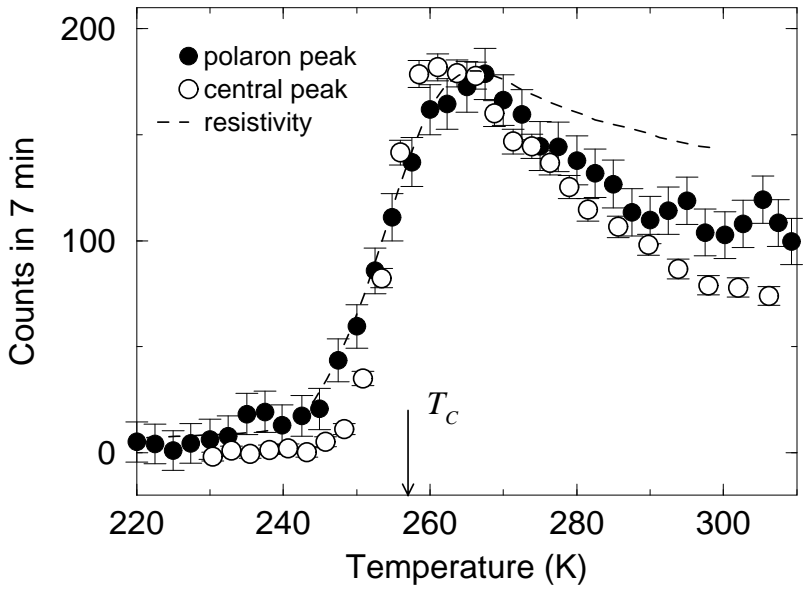

FIG. 4. Temperature dependence of the intensity of the polaron peak at $(3.75,0.25,0)$, compared to the central peak scattering at a wave vector of $(1.03,0,0)$. The data have been scaled so the peak heights match; the count rate describes the charge peak. The dashed curve is the resistivity, scaled in the same manner. The similarity of the data indicate a common physical origin.

The above experimental results reveal that both the spin and charge correlations associated with the polarons in $\mathrm{La}_{0.7} \mathrm{Ca}_{0.3} \mathrm{MnO}_{3}$ appear together, and have a very similar spatial and temperature dependence. The metalinsulator crossover in the conductivity also occurs close to $T_{C}$. This coincidence may explain the amplified magnetoresistive effects, as well as the absence of conventional magnetic critical behavior, both in the Ca-doped system as well as in other materials [7]. This behavior is not universal, however. In the higher $T_{C} \mathrm{Sr}$ and Ba systems, for example, the polaron formation [5,8,20] and conductivity crossover [21] can occur at temperatures substantially higher than the Curie point, reducing the magnetoresistive effects and rendering the spin dynamics more conventional. The physical quantities that control the characteristic temperature for polaron formation, including the role that metallurgical defects play in their formation, still remain to be elucidated.

The work at UM was supported by the NSF, DMR 9701339, and NSF-MRSEC, DMR 00-80008. The work in Moscow was supported by ISTC grant \#636.

[1] G.H. Jonker and J.H. Van Santen, Physica 16, 337 (1950); ibid 19, 120 (1950); E.O. Wollan and W.C. Koehler, Phys. Rev. 100, 545 (1955); P. Schiffer, A.P. Ramirez, W. Bao, and S-W. Cheong, Phys. Rev. Lett. 75, 3336 (1995). For a recent experimental review see A.P. Ramirez, J. Phys. Cond. Matter 9, 8171 (1997).

[2] C. Zener, Phys. Rev. 82, 403 (1951); P.W. Anderson and H. Hasegawa, ibid. 100, 675 (1955); P.G. de Gennes, ibid.
118, 141 (1960); J.B. Goodenough, ibid. 100, 564 (1955).

[3] For some recent theoretical work see A.J. Millis, P.B. Littlewood, and B.I. Shraiman, Phys. Rev. Lett. 74, 5144 (1995); H. Röder, J. Zang, and A.R. Bishop, ibid. 76, 1356 (1996); A.J. Millis, Phys. Rev. B 53, 8434 (1996); C. M. Varma, Phys. Rev. B 54, 7328 (1996); A.S. Alexandrov and A.M. Bratkovsky, Phys. Rev. Lett. 82, 141 (1999); A. Moreo, S. Yunoki, and E. Dagotto, Science 283, 2034 (1999).

[4] H.Y. Hwang, P. Dai, S-W. Cheong, G. Aeppli, D.A. Tennant, and H.A. Mook, Phys. Rev. Lett. 80, 1316 (1998).

[5] L. Vasiliu-Doloc, J.W. Lynn, A.H. Moudden, A.M. de Leon-Guevara, and A. Revcolevschi, Phys. Rev. B 58, 14913 (1998)

[6] J.W. Lynn, R.W. Erwin, J.A. Borchers, Q. Huang, A. Santoro, J-L. Peng, and Z.Y. Li, Phys. Rev. Lett. 76, 4046 (1996); J.W. Lynn, R.W. Erwin, J.A. Borchers, A. Santoro, Q. Huang, J-L. Peng, and R. L. Greene, J. Appl. Phys. 81, 5488 (1997).

[7] J.A. Fernandez-Baca, P. Dai, H.Y. Hwang, C. Kloc, and S-W. Cheong, Phys. Rev. Lett. 80, 4012 (1998).

[8] L. Vasiliu-Doloc, J.W. Lynn, A.H. Moudden, A.M. de Leon-Guevara, and A. Revcolevschi, J. Appl. Phys. 81, 5491 (1997).

[9] S.J.L. Billinge, R.G. DiFrancesco, G.H. Kwei, J. J. Neumeier, and J.D. Thompson, Phys. Rev. Lett. 77, 715 (1996); D. Louca, T. Egami, E.L. Brosha, H. Röder, and A.R. Bishop, Phys. Rev. B 56, 8475 (1997).

[10] J.M. deTeresa, M.R. Ibarra, P.A. Algarabel, C. Ritter, C. Marquina, J. Blasco, J. Garcia, A. del Moral, and Z. Arnold, Nature 386, 256 (1997).

[11] For a review, see A.S. Alexandrov and N.F. Mott, Polarons and Bipolarons (World Scientific, Singapore, 1995).

[12] L. Vasiliu-Doloc, S. Rosenkranz, R. Osborn, S.K. Sinha, J.W. Lynn, J. Mesot, O.H. Seeck, G. Presosti, A.J. Fedro, and J.F. Mitchell, Phys. Rev. Lett. 83, 4393 (1999).

[13] S. Shimomura, N. Wakabayashi, H. Kuwahara, and Y. Tokura, Phys. Rev. Lett. 83, 4389 (1999).

[14] D. Shulyatev, S. Karabashev, A. Arsenov, and Y. Mukovskii, J. Cryst. Growth 199, 511 (1999).

[15] For a review see J.W. Lynn, J. Superconductivity and Novel Magnetism 13, 263 (2000).

[16] G.M. Zhao, K. Conder, H. Keller, and K.A. Müller, Nature, (London) 381, 676 (1996).

[17] S. Mori, C.H. Chen, and S-W. Cheong, Nature (London) 392, 473 (1998); S. Mori, C.H. Chen, and S-W. Cheong, Phys. Rev. Lett. 81, 3972 (1998).

[18] J.M. Tranquada, D.J. Buttrey, V. Sachan, and J.E. Lorenzo, Phys. Rev. Lett. 73, 1003 (1994).

[19] S.-H. Lee and S-W. Cheong, Phys. Rev. Lett. 792514 (1997).

[20] J.W. Lynn, L. Vasiliu-Doloc, K. Ghosh, G-m. Zhao, V. Smolyaninova, R.L. Greene, S. N. Barilo, G. L. Bychkov, and L.A. Kurnevitch, (preprint).

[21] A. Urushibara, Y. Moritomo, T. Arima, A. Asamitsu, G. Kido, and Y. Tokura, Phys. Rev. B 51, 14103 (1995); A. Anane, C. Dupas, K. Le Dang, J.P. Renard, P. Veillet, A.M. de Leon-Guevara, F. Millot, L. Pinsard, and A. Revcolevschi, J. Phys.: Condens. Matter 7, 7015 (1995). 